\title{
A STRUCTURE-PRESERVING NUMERICAL DISCRETIZATION OF REVERSIBLE DIFFUSIONS*
}

\author{
JUAN C. LATORRE ${ }^{\dagger}$, PHILIPP METZNER ${ }^{\ddagger}$, CARSTEN HARTMANN $^{\S}$, \\ AND CHRISTOF SCHÜTTEף
}

\begin{abstract}
We propose a numerical discretization scheme for the infinitesimal generator of a diffusion process based on a finite volume approximation. The resulting discrete-space operator can be interpreted as a jump process on the mesh whose invariant distribution is precisely the cell approximation of the Boltzmann invariant measure and preserves the detailed balance property of the original stochastic process. Moreover this approximation is robust in the sense that these properties remain valid independently of the grid size.
\end{abstract}

Key words. Reversible diffusions, finite-volume method, detailed balance, coarse-graining.

AMS subject classifications. 60G10, 60H35, 60J27, 65N08.

\section{Introduction and overview}

In many applications, e.g., molecular dynamics [39], reaction kinetics [16], or systems biology [1], one is interested in discrete-state approximations of the following class of stochastic differential equations (SDE):

$$
d X_{t}=-\nabla V\left(X_{t}\right) d t+\sqrt{2 \beta^{-1}} d W_{t}, \quad X_{0}=x .
$$

Here $W_{t}$ denotes standard Brownian motion on the state space of $X_{t}$, the function $V$ is a smooth potential, and $\beta=1 /\left(k_{B} T\right)$ denotes inverse temperature with $k_{B}$ being Boltzmann's constant (more precise statements are given below).

It is desirable that the numerical approximation of the stochastic process inherits some of the basic properties of the original equation such as its stationary distribution or reversibility. The latter is equivalent to the requirement that the discretized process satisfies detailed balance, which essentially asserts that the probability fluxes between equilibrium states are balanced [15]; therefore any sensible discretization method for the problem at hand should be targeted on approximating the corresponding fluxes (this excludes, e.g., finite differences or finite elements approximations).

A popular method in computational fluid dynamics is the finite volume method as it is based on evaluating fluxes through the surfaces of the discretization volumes [43]. Moreover the method is conservative in the sense that the flux entering a certain volume is equal to the flux coming from the neighboring volumes [26]. The flux conservation property entails that there is no loss of probability at the boundaries of the computational domains; see the related works [6] or [2] for a discussion of the Discontinuous Galerkin method.

${ }^{*}$ Received: April 20, 2010; accepted (in revised version): March 20, 2011. Communicated by Eric Vander Eijnden.

${ }^{\dagger}$ To whom correspondence should be addressed: Freie Universität Berlin, Institut für Mathematik, Arnimallee 6, 14195 Berlin, Germany (latorre@mi.fu-berlin.de).

${ }^{\ddagger}$ Freie Universität Berlin, Institut für Mathematik, Arnimallee 6, 14195 Berlin, Germany (metzner @mi.fu-berlin.de).

${ }^{\S}$ Freie Universität Berlin, Institut für Mathematik, Arnimallee 6, 14195 Berlin, Germany (chartman@mi.fu-berlin.de).

『Freie Universität Berlin, Institut für Mathematik, Arnimallee 6, 14195 Berlin, Germany (schuette@mi.fu-berlin.de). 
In this paper we follow ideas of Wang, Peskin, and Elston [42], where a detailed balance preserving numerical algorithm is developed for the study of Brownian motors, and further work [24, 23] where the authors study Dirichlet problems for the computation of mean exit times in the low temperature regime (cf. Section 3.2). We present a numerical approximation of the second-order differential operator

$$
\mathcal{L}=\beta^{-1} \Delta-\nabla V(x) \cdot \nabla
$$

that is associated with the diffusion (1.1) and that generates the semigroup $\exp (t \mathcal{L})$. More precisely, we are interested in studying elliptic boundary value problems

$$
\begin{aligned}
\mathcal{L} u=f & & x \in \Omega, \\
\mathcal{B} u+\mathcal{C} \nabla u=g & & x \in \partial \Omega,
\end{aligned}
$$

for given data $f$ and $g$ where $\Omega \subset \mathbb{R}^{n}$ is an open subset of $\mathbb{R}^{n}$ with smooth (say, piecewise continuous) boundary $\partial \Omega$, and $\mathcal{B}, \mathcal{C}$ are trace operators. Moreover we will discuss parabolic equations of the form

$$
\begin{aligned}
\left(\frac{\partial}{\partial t}-\mathcal{L}^{*}\right) \rho & =0 & & (x, t) \in \Omega \times(0, \infty), \\
\rho & =\rho_{0} & (x, t) & \in \Omega \times\{0\},
\end{aligned}
$$

where $\mathcal{L}^{*}$ is the formal $L^{2}$ adjoint of $\mathcal{L}$, namely,

$$
\mathcal{L}^{*}=\beta^{-1} \Delta+\nabla V(x) \cdot \nabla+\Delta V(x) .
$$

If we assume that $V \in C^{\infty}(\Omega)$ is bounded below and satisfies the usual growth conditions for $|\Omega| \rightarrow \infty$, then $\rho(x, t)=\exp \left(t \mathcal{L}^{*}\right) \rho_{0}(x)$ equals the probability distribution

$$
\rho(x, t) d x=\mathbb{P}\left[X_{t} \in[x, x+d x)\right]
$$

of $X_{t}$ given that $X_{0}$ follows an initial distribution $\rho_{0}$. There are several requirements that the discretization of $\mathcal{L}$ or $\mathcal{L}^{*}$ should fulfill:

1. The spatial discretization of the Boltzmann distribution

$$
d \mu(x)=Z^{-1} e^{-\beta V(x)} d x, \quad Z=\int_{\Omega} e^{-\beta V(x)} d x,
$$

that is, the stationary distribution of (1.1), should equal the stationary solution of the discretized Fokker-Planck Equation (1.4). In particular, given a uniform discretization $x_{1}, x_{2}, \ldots, x_{M}$ of $\Omega$, we require $\boldsymbol{\pi}=\left(\pi_{1}, \ldots, \pi_{M}\right)$ with $\pi_{i}=\exp \left(-\beta V\left(x_{i}\right)\right)$ to be the stationary solution of (1.4), upon discretization and up to a (constant) normalization factor.

2. The discretization should preserve the forward backward dichotomy, i.e., when $\boldsymbol{A}$ denotes the spatial discretization of the infinitesimal generator $\mathcal{L}$, then we want $\boldsymbol{A}^{T}$ to be the discretization of its adjoint $\mathcal{L}^{*}$ (including boundary conditions).

3. Being related with the latter, we require that the discretization preserves detailed balance. That is, for all $(x, y) \in \Omega$ and $t \in[0, T]$ the transition probabilities satisfy

$$
\mathbb{P}\left[X_{t} \in[x, x+d x) \mid X_{0}=y\right] d \mu(y)=\mathbb{P}\left[X_{t} \in[y, y+d y) \mid X_{0}=x\right] d \mu(x),
$$


which implies that the time-reversed process $\tilde{X}_{t}=X_{T-t}$ with stationary initial condition $X_{0} \sim \mu$ follows the same SDE as the original process, hence has the same infinitesimal generator (cf. $[15,18]$ ).

In [42] the problem stated above is approached as follows. From the original SDE (1.1), the authors find the coefficients (transition rates) of a Markov jump process (MJP) by balancing the fluxes between adjacent grid points such that the resulting discrete-state process has the correct invariant distribution (the cell approximation of the Boltzmann invariant measure) and satisfies the detailed balance condition. In this paper we wish to extend these ideas by rigorously approximating $\mathcal{L}$ and, likewise, $\mathcal{L}^{*}$ using a finite volume method. In doing so, the discrete approximation of the infinitesimal generator $\mathcal{L}$ not only results in the infinitesimal generator $\boldsymbol{A}$ of a MJP with the correct approximation of the invariant distribution of (1.1) and detailed balance, but also the discretization of the adjoint operator $\mathcal{L}^{*}$ results precisely in the adjoint of the discrete generator $\boldsymbol{A}$, which is an essential property for applying existing methods for finding transition paths and analyzing rare events in metastable systems (see Section 3.2). It can be moreover shown that the transition rates of the MJP found in [42] are, in fact, a further approximation of the transition rates given by (2.8) found using the finite volume method. The finite volume method can be applied seamlessly to multi-dimensional systems and provides the means for using non-rectangular cell geometries as well as computing error estimates, making possible the study of problems with complex boundary geometries and the use of mesh-refinement methods (see Sections 2.4 and 4). Finally, this approach allows us to consistently apply averaging methods for SDE's and MJP's in order to reduce the number of dimensions of the system whenever this is possible (see Section 4).

The article is organized as follows: in Section 2 we derive the structure-preserving finite volume discretization of the infinitesimal generator that gives rise to discretizations of the forward equation (Section 2.1) and the backward equation (Section 2.2). Section 3 contains a couple of numerical examples, both time-dependent and timeindependent. We conclude by a brief discussion of the results in Section 4.

\section{Finite volume approximation of the infinitesimal generator}

In this section we derive a spatial discretization of the SDE (1.1) based on a finite volume approximation $[25,26]$ of the infinitesimal generator and its adjoint. To this end, it is convenient to recast the infinitesimal generator (1.2) as

$$
\mathcal{L} u=\beta^{-1} e^{\beta V} \nabla \cdot\left(e^{-\beta V} \nabla u\right),
$$

and its adjoint as

$$
\mathcal{L}^{*} \rho=\beta^{-1} \nabla \cdot\left(e^{-\beta V} \nabla\left(e^{\beta V} \rho\right)\right) .
$$

Use of symbols and standing assumptions. Before we derive the discretizations of $\mathcal{L}$ and $\mathcal{L}^{*}$, we shall fix some notation that we will use in the following. We call (see Figure 2.1)

- $x_{i} \in \Omega$ : mesh point $i, i=1, \ldots, M$; the discrete state space is denoted by $S$.

- $\Omega_{i}$ : finite volume element (cell) of mesh point $i \in S$,

- $m\left(\Omega_{i}\right)$ : Lebesgue measure (volume) of element $\Omega_{i}$,

- for every $i \in S$, we denote by $\left\{i_{l}\right\}, l=1, \ldots, M_{i}$ the subsequence, such that the $\left\{x_{i_{l}}\right\}$ are neighbors (adjacent mesh points) of $x_{i}$, 
- $S_{i, j}$ : boundary (plane segment) between $\Omega_{i}$ and $\Omega_{j}$ for $j \in\left\{i_{l}\right\}$,

- $h_{i, j}$ : line segment connecting $x_{i}$ and $x_{j}$ for $j \in\left\{i_{l}\right\}$,

- $\bar{x}_{i, j}: S_{i, j} \cap h_{i, j}$ for $j \in\left\{i_{l}\right\}$.

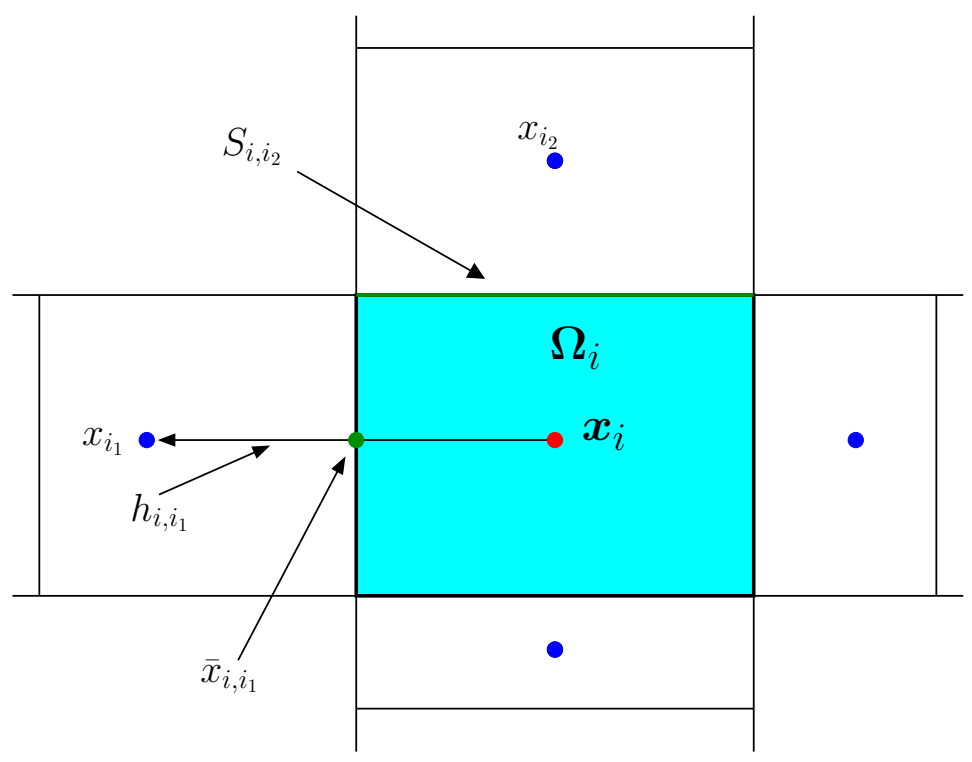

Figure 2.1. Finite-volume element $\Omega_{i}$.

We assume throughout that the cell elements $\Omega_{i}$ are rectangular. Accordingly, any vector $h_{i, j}$ connecting two neighboring points $x_{i}$ and $x_{j}$ is perpendicular to the surface element $S_{i, j}$ dividing the neighboring cells. Note, however, that this does not imply that the mesh is uniform along the principal coordinate directions.

2.1. Numerical discretization of the forward equation. We firstly confine our attention to the forward Kolmogorov (Fokker-Planck) equation. To this end we use (2.2) and rewrite the forward Equation (1.4) as

$$
\frac{\partial}{\partial t} \rho(x, t)=\beta^{-1} \nabla \cdot\left(e^{-\beta V(x)} \nabla\left(e^{\beta V(x)} \rho(x, t)\right)\right) .
$$

Now given a set of discretization points $\left\{x_{i}\right\}_{i=1}^{M} \subset \Omega$, we associate a finite volume element $\Omega_{i}$ to each point $x_{i} \in \Omega_{i}$ and introduce

$$
p_{i}(t)=\int_{\Omega_{i}} \rho(x, t) d x
$$

as the homogeneous probability distribution of $X_{t}$ on the cell $\Omega_{i}$. Using the divergence theorem it follows that

$$
\begin{aligned}
\frac{d}{d t} p_{i}(t) & =\int_{\Omega_{i}} \beta^{-1} \nabla \cdot\left(e^{-\beta V(x)} \nabla\left(e^{\beta V(x)} \rho\right)\right) d x \\
& =\sum_{j \in\left\{i_{l}\right\}} \int_{S_{i, j}} \beta^{-1} e^{-\beta V(x)} \nabla\left(e^{\beta V(x)} \rho\right) \cdot \boldsymbol{n} d s .
\end{aligned}
$$


For all $j \in\left\{i_{l}\right\}$, we approximate each surface integral above as a point evaluation of the integrand at the middle point $\bar{x}_{i, j}$ times the area of $S_{i, j}$,

$$
\begin{aligned}
& \int_{S_{i, j}} \beta^{-1} e^{-\beta V(x)} \nabla\left(e^{\beta V(x)} \rho\right) \cdot \boldsymbol{n} d s \\
\approx & \left.\beta^{-1} e^{-\beta V\left(\bar{x}_{i, j}\right)} \nabla\left(e^{\beta V(x) \rho}\right) \cdot \boldsymbol{n}\right|_{x=\bar{x}_{i, j}} m\left(S_{i, j}\right) .
\end{aligned}
$$

As we assume that the vector $h_{i, j}$ is parallel to the unit normal $\boldsymbol{n}$ to the surface $S_{i, j}$ dividing the cells $\Omega_{i}$ and $\Omega_{j}$, we can write

$$
\left.\nabla f \cdot \boldsymbol{n}\right|_{x=\bar{x}_{i, j}}=\left.\frac{\nabla f \cdot h_{i, j}}{m\left(h_{i, j}\right)}\right|_{x=\bar{x}_{i, j}}, \quad j \in\left\{i_{l}\right\}
$$

for any differentiable function $f$, where $m\left(h_{i, j}\right)$ here is just the length of the line $h_{i, j}$. But the last expression is simply the directional derivative of $f$ at $\bar{x}_{i, j}$. Hence, using a two-sided finite difference approximation between the points $x_{i}$ and $x_{j}$, we obtain

$$
\left.\nabla\left(e^{\beta V(x)} \rho\right) \cdot \boldsymbol{n}\right|_{x=\bar{x}_{i, j}} \approx \frac{e^{\beta V_{j}} \rho\left(x_{j}, t\right)-e^{\beta V_{i}} \rho\left(x_{i}, t\right)}{m\left(h_{i, j}\right)},
$$

where for simplicity we have used the shorthand $V_{k}=V\left(x_{k}\right)$. Upon employing the approximation

$$
p_{i}(t)=\int_{\Omega_{i}} \rho(x, t) d x \approx \rho\left(x_{i}, t\right) m\left(\Omega_{i}\right),
$$

the probability flux on $S_{i, j}$ can be recast as

$$
\begin{aligned}
& \int_{S_{i, j}} \beta^{-1} e^{-\beta V(x)} \nabla\left(e^{\beta V(x)} \rho\right) \cdot \boldsymbol{n} d s \\
\approx & \frac{e^{-\beta\left(V_{i, j}-V_{j}\right)}}{\Delta_{j, i}} p_{j}(t)-\frac{e^{-\beta\left(V_{i, j}-V_{i}\right)}}{\Delta_{i, j}} p_{i}(t), \quad j \in\left\{i_{l}\right\},
\end{aligned}
$$

where $V_{i, j}=V\left(\bar{x}_{i, j}\right)$ and

$$
\frac{1}{\Delta_{i, j}}=\frac{\beta^{-1} m\left(S_{i, j}\right)}{m\left(h_{i, j}\right) m\left(\Omega_{i}\right)}, \quad j \in\left\{i_{l}\right\} .
$$

Regrouping terms in (2.3), the approximation of (1.4) finally becomes

$$
\frac{d}{d t} p_{i}(t)=\sum_{j \in\left\{i_{l}\right\}} \frac{e^{-\beta\left(V_{i, j}-V_{j}\right)}}{\Delta_{j, i}} p_{j}(t)-\left(\sum_{j \in\left\{i_{l}\right\}} \frac{e^{-\beta\left(V_{i, j}-V_{i}\right)}}{\Delta_{i, j}}\right) p_{i}(t)
$$

or, in matrix vector notation,

$$
\dot{\boldsymbol{p}}(t)=\boldsymbol{A}^{T} \boldsymbol{p}(t)
$$

with $\boldsymbol{p}=\left(p_{1}, \ldots, p_{M}\right)^{T}$. Here and in the following dotted quantities such as $\dot{\boldsymbol{p}}=d \boldsymbol{p} / d t$ denote time derivatives and the elements of the matrix $\boldsymbol{A} \in \mathbb{R}^{M \times M}$ are given by

$$
A_{i, j}= \begin{cases}\Delta_{i, j}^{-1} e^{-\beta\left(V_{i, j}-V_{i}\right)}, & j \in\left\{i_{l}\right\} \\ -\sum_{k \in\left\{i_{l}\right\}} A_{i, k}, & j=i, \\ 0, & \text { otherwise. }\end{cases}
$$


The matrix $\boldsymbol{A}$ has row sum zero and off-diagonal entries that are non-negative. Hence it has all properties of a generator matrix. In more specific terms, $\boldsymbol{A}$ is the infinitesimal generator of a jump process on the grid $\left\{x_{i}\right\}$ with the $A_{i, j}$ for $i \neq j$ being the jump rates between the discrete states $x_{i}$ and $x_{j}$.

2.2. Finite-volume approximation of the backward equation. Next, we derive the finite volume approximation of the generator $\mathcal{L}$ in terms of the backward Kolmogorov equation that is the adjoint to (1.4). It turns out that the discretization of $\mathcal{L}$ is precisely the discrete operator $\boldsymbol{A}$ from the previous section; note that this is not generally the case for any given spatial discretization method (e.g., finite differences).

Using the representation $(2.1)$ of the generator $\mathcal{L}$, we proceed. As before, the backward Kolmogorov equation reads

$$
\frac{\partial}{\partial t} u(x, t)=\beta^{-1} e^{\beta V(x)} \nabla \cdot\left(e^{-\beta V(x)} \nabla u(x, t)\right) .
$$

Setting $u_{i}(t)=u\left(x_{i}, t\right)$ and integrating $(2.9)$ over the cell element $\Omega_{i}$, we find

$$
\begin{aligned}
\frac{d}{d t} u_{i}(t) m\left(\Omega_{i}\right) & \approx \int_{\Omega_{i}} \beta^{-1} e^{\beta V(x)} \nabla \cdot\left(e^{-\beta V(x)} \nabla u\right) \\
& \left.\approx \beta^{-1} e^{\beta V\left(x_{i}\right)} \nabla \cdot\left(e^{-\beta V(x)} \nabla u\right)\right|_{x=x_{i}} m\left(\Omega_{i}\right),
\end{aligned}
$$

where in the first equality we have made the approximation

$$
\frac{d}{d t} u_{i}(t) m\left(\Omega_{i}\right) \approx \frac{d}{d t} \int_{\Omega_{i}} u(x, t) d x
$$

and we have again approximated the volume integral as a point evaluation at $x=x_{i}$ times the volume of $\Omega_{i}$. Doing a backward substitution, the divergence term in the above equation can be rewritten as

$$
\begin{aligned}
\left.\nabla \cdot\left(e^{-\beta V(x)} \nabla u\right)\right|_{x=x_{i}} m\left(\Omega_{i}\right) & \approx \int_{\Omega_{i}} \nabla \cdot\left(e^{-\beta V(x)} \nabla u\right) d x \\
& =\sum_{j \in\left\{i_{l}\right\}} \int_{S_{i, j}} e^{-\beta V(x)} \nabla u \cdot \boldsymbol{n} d s .
\end{aligned}
$$

where the second line follows again from the divergence term. For each individual surface integral over $S_{i, j}, j \in\left\{i_{l}\right\}$ we may then write

$$
\begin{aligned}
\int_{S_{i, j}} e^{-\beta V(x)} \nabla u \cdot \boldsymbol{n} d s & \left.\approx e^{-\beta V(x)} \nabla u \cdot \boldsymbol{n}\right|_{x=\bar{x}_{i, j}} m\left(S_{i, j}\right) \\
& =\left.e^{-\beta V_{i, j}} \frac{\nabla u \cdot h_{i, j}}{m\left(h_{i, j}\right)}\right|_{x=\bar{x}_{i, j}} m\left(S_{i, j}\right) \\
& \approx e^{-\beta V_{i, j}} \frac{u\left(x_{j}, \cdot\right)-u\left(x_{i}, \cdot\right)}{m\left(h_{i, j}\right)} m\left(S_{i, j}\right) \\
& =\frac{e^{-\beta V_{i, j}} m\left(S_{i, j}\right)}{m\left(h_{i, j}\right)} u_{j}-\frac{e^{-\beta V_{i, j}} m\left(S_{i, j}\right)}{m\left(h_{i, j}\right)} u_{i} .
\end{aligned}
$$

As we did for the forward Kolmogorov equation, the surface integral has been approximated as a point evaluation of the integrand at $x=\bar{x}_{i, j}$, while the resulting normal 
derivative was replaced by a centered difference at the points $x=x_{i}$ and $x=x_{j}$. After regrouping terms in (2.10), we obtain

$$
\begin{aligned}
\frac{d}{d t} u_{i}(t) & =\sum_{j \in\left\{i_{l}\right\}} \frac{e^{-\beta\left(V_{i, j}-V_{i}\right)}}{\Delta_{i, j}} u_{j}(t)-\sum_{j \in\left\{i_{l}\right\}} \frac{e^{-\beta\left(V_{i, j}-V_{i}\right)}}{\Delta_{i, j}} u_{i}(t) \\
& =\sum_{j \in\left\{i_{l}\right\}} A_{i, j} u_{j}(t)-\left(\sum_{j \in\left\{i_{l}\right\}} A_{i, j}\right) u_{i}(t)
\end{aligned}
$$

or, in matrix vector notation,

$$
\dot{\boldsymbol{u}}(t)=\boldsymbol{A u}(t),
$$

where $A_{i, j}$ is precisely given by (2.8), thus proving that the finite volume approximation (FVA) of the backward Kolmogorov equation results in the adjoint equation of the forward Kolmogorov (master) Equation (2.7) for the discrete-state system.

\subsection{Properties of the semi-discretized Kolmogorov equations.}

1. Recall that the Boltzmann distribution

$$
\mu(d x)=Z^{-1} e^{-\beta V(x)} d x, \quad Z=\int_{\Omega} e^{-\beta V(x)} d x
$$

is the unique invariant distribution of the continuous state space process (1.1). On the other hand the homogeneous probability distribution of the process on the discretized state space $S$ reads

$$
\begin{aligned}
\mathbb{P}\left[X \in \Omega_{i}\right] & =\int_{\Omega_{i}} d \mu(x) \\
& \approx Z^{-1} e^{-\beta V_{i}} m\left(\Omega_{i}\right) .
\end{aligned}
$$

It can be readily seen that the vector

$$
\boldsymbol{\pi}=\left(\pi_{1}, \ldots, \pi_{M}\right), \quad \pi_{i} \propto e^{-\beta V_{i}} m\left(\Omega_{i}\right) .
$$

is indeed a solution to the stationary Fokker-Planck equation for the discretestate system, i.e., $\boldsymbol{A}^{T} \boldsymbol{\pi}=0$. To see this, we substitute $\boldsymbol{\pi}$ into (2.6):

$$
\begin{aligned}
{\left[\boldsymbol{A}^{T} \boldsymbol{\pi}\right]_{i} } & =\sum_{j \in\left\{i_{l}\right\}} \frac{e^{-\beta\left(V_{i, j}-V_{j}\right)}}{\Delta_{j, i}} e^{-\beta V_{j}} m\left(\Omega_{j}\right)-\sum_{j \in\left\{i_{l}\right\}} \frac{e^{-\beta\left(V_{i, j}-V_{i}\right)}}{\Delta_{i, j}} e^{-\beta V_{i}} m\left(\Omega_{i}\right) \\
& =\sum_{j \in\left\{i_{l}\right\}} \frac{e^{-\beta\left(V_{i, j}\right)}}{\Delta_{j, i}} m\left(\Omega_{j}\right)-\sum_{j \in\left\{i_{l}\right\}} \frac{e^{-\beta\left(V_{i, j}\right)}}{\Delta_{i, j}} m\left(\Omega_{i}\right) \\
& =\sum_{j \in\left\{i_{l}\right\}} \frac{\beta^{-1} m\left(S_{i, j}\right)}{m\left(h_{j, i}\right)} e^{-\beta\left(V_{i, j}\right)}-\sum_{j \in\left\{i_{l}\right\}} \frac{\beta^{-1} m\left(S_{i, j}\right)}{m\left(h_{i, j}\right)} e^{-\beta\left(V_{i, j}\right)} \\
& =0
\end{aligned}
$$

where we have used $\bar{x}_{i, j}=\bar{x}_{j, i}, m\left(S_{i, j}\right)=m\left(S_{j, i}\right)$ and $m\left(h_{i, j}\right)=m\left(h_{j, i}\right), j \in$ $\left\{i_{l}\right\}$. Hence (2.13) is a stationary distribution of (2.7). In particular, the restriction of $\exp (-\beta V)$ to the grid points is a stationary distribution if the grid is uniform. 
2. It is an immediate consequence of (2.7) and (2.12) that the diagram

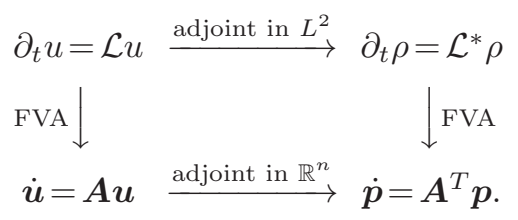

commutes. Hence the finite volume approximation (FVA) yields adjointness of the discrete backward and forward equations.

3. Last but not least, the discretization preserves detailed balance, i.e., reversibility of the original process (i.e., $X_{t}$ and $X_{T-t}$ have the same generator). For a jump process with infinitesimal generator $\boldsymbol{A}$, the detailed balance condition reads

$$
A_{i, j} \pi_{i}=A_{j, i} \pi_{j} \quad \forall i, j \in S .
$$

For our jump process with master Equation (2.6) the last equation holds true since $A_{i, j}=A_{j, i}=0$ for $j \notin\left\{i_{l}\right\}$, which immediately implies $A_{i, j} \pi_{i}=A_{j, i} \pi_{j}$; for $j \in\left\{i_{l}\right\}$, we have

$$
\begin{aligned}
\pi_{i} A_{i, j} & =e^{-\beta V_{i}} m\left(\Omega_{i}\right) \frac{e^{-\beta\left(V_{i, i_{l}}-V_{i}\right)}}{\Delta_{i, i_{l}}} \\
& =\frac{\beta^{-1} m\left(S_{i, i_{l}}\right)}{m\left(h_{i, i_{l}}\right)} e^{-\beta V_{i, i_{l}}},
\end{aligned}
$$

while

$$
\begin{aligned}
\pi_{j} A_{j, i} & =e^{-\beta V_{i_{l}}} m\left(\Omega_{i_{l}}\right) \frac{e^{-\beta\left(V_{i_{l}, i}-V_{i_{l}}\right)}}{\Delta_{i_{l}, i}} \\
& =\frac{\beta^{-1} m\left(S_{i, i_{l}}\right)}{m\left(h_{i, i_{l}}\right)} e^{-\beta V_{i, i_{l}}},
\end{aligned}
$$

which proves the assertion that the discrete system is again reversible.

We would like to emphasize that these properties are satisfied independently of the size of the cell elements ${ }^{1}$. In other words, the discrete operator $\boldsymbol{A}$ is the infinitesimal generator of a jump process independently of the size of the cell elements. Moreover its invariant distribution is given by (2.13) and it satisfies the detailed balance condition with respect to this distribution.

2.4. On the accuracy of the method and non-rectangular grids. Before we conclude this section we would like to briefly elaborate on the order of accuracy of finite volume approximation as well as comment on the assumptions underlying the definition of the cell elements.

Assuming a uniform mesh, every cell has the same length $h$ in each spatialdirection. Hence the finite volume approximation of the special backward generator (2.1) is equivalent to using centered finite differences at each mesh point $x_{i}$. To be more precise, for the divergence term in (2.1) one can use a centered finite difference between the points $x_{i+1 / 2}$ and $x_{i-1 / 2}$ (for each coordinate direction in $\Omega$ ), resulting

\footnotetext{
${ }^{1}$ Without concerns regarding the accuracy or stability of the approximation.
} 
in the evaluation of the potential at the "mid-points" $\bar{x}_{i_{l}}$. The gradient $\nabla u$ at $\bar{x}_{i_{l}}$ can then be approximated by a centered finite difference between the points $x_{i}$ and $x_{i \pm 1}$ as before. This will result in the discrete state approximation (2.8) of the infinitesimal generator $\mathcal{L}$. Given these simplifying assumptions and assuming some regularity of the potential $V$, the finite volume discretization (2.8) turns out to be a consistent approximation of the infinitesimal generator (1.2) that is second-order accurate $[7$, $40,26]$. This assertion will be verified numerically in Section 3.1 where we show the solution of Dirichlet problems of the type $\mathcal{L} u=f$. In [42], where a similar numerical scheme is considered, it is argued that the numerical algorithm is robust even for potentials that may have discontinuities. In Section 3 we present more details on how the boundary conditions are seamlessly incorporated into the method in the context of the numerical examples.

In the derivation of the numerical algorithm we have assumed that the cell elements are $n$-dimensional hyperrectangles and that, along each direction, the neighboring points of each mesh point are connected by a vector normal to the cell dividing surfaces. This hypothesis can be relaxed; in fact, each cell element may have any arbitrary convex polygonal as long as condition (2.5) is satisfied. This condition states that the line segment connecting two neighboring mesh points in perpendicular to the hyperplane dividing their respective cells. Under these circumstances the derivation of the method follows exactly as it was done throughout this section, while preserving all the aforementioned properties (see Section 4 for further comments on this matter). If condition (2.5) is not satisfied, one could consider cells with arbitrary convex polygonal shape without affecting the overall method - probably even without affecting reversibility or the forward-backward dichotomy, but we could no longer approximate the probability flux along each boundary as in (2.4) simply using finite differences; as a consequence the scheme would not admit an expression for the discrete generator as simple as (2.8), but rather require the computation of certain surface integrals which may be tedious if the problem becomes high-dimensional (cf. also [3] and the references therein). There are clearly other methods to approximate fluxes in a finite volume setting (see e.g. [11]), but these may not result in the desired form of the infinitesimal generator.

\section{Numerical experiments}

In this section we illustrate the finite volume approximation (FVA) with several test problems. The first example is concerned with solving mixed boundary value problems involving the backward generator $\mathcal{L}$. In the second and third example we demonstrate several properties of the FVA, which go beyond standard applications by exploiting the fact that the discretization matrix $\boldsymbol{A}$ can be interpreted as an infinitesimal generator of a MJP exhibiting the structural and dynamical properties of the original Markov diffusion process. In the second example we apply the FVA to rare events problems and study the rearrangement of a three-particle Lennard-Jones cluster in the plane. Finally, in the third example we show how the FVA can be used to sample the original stochastic dynamics (1.1) in cases when the direct numerical simulation (DNS) is not feasible, e.g., when the system is highly metastable so that the time discretization errors accumulate and thus bias the stationary distribution (if it exists at all).

3.1. Committor for the two-well potential. The evolution of statistical objects associated with stochastic processes of the form (1.1) such as conditional expectations or initial probability densities are governed by the backward generator $\mathcal{L}$ and its adjoint $\mathcal{L}^{*}$. Computing those objects amounts to solving partial differen- 
tial equations involving these operators with a set of problem-dependent boundary conditions. In first example we apply FVA to a class of mixed boundary value problems arising in the analysis of rare events of Markov diffusion processes, particularly in Transition Path Theory (TPT). We shall come back to TPT type problems in Section 3.2.

The mixed boundary value problems are of the type

$$
\left\{\begin{aligned}
\mathcal{L} q=0 & \text { in } \Omega, \\
q=0 & \text { on } \partial A, \\
q=1 & \text { on } \partial B, \\
\nabla q \cdot \boldsymbol{n}=0 & \text { on } \partial \Omega,
\end{aligned}\right.
$$

where $\mathcal{L}$ is the backward generator given in (1.2), $\Omega \subset \mathbb{R}^{n}$ is a compact set with smooth boundary $\partial \Omega$, the sets $A, B \subset \Omega$ are open, disjoint subsets with smooth boundaries $\partial A, \partial B$, and $\boldsymbol{n}$ denotes the outward pointing unit normal to $\partial \Omega$. The solution of the problem (3.1) is called forward committor function and is the key object in TPT.

Before we explain our numerical experiment in detail, we would like to briefly comment on the standard numerical methods to which the FVA is compared. The guiding property for selecting standard methods for the comparison is the neighborhood relation of the boxes involved in the discretization stencil of the FVA. As one can see from the definition of the discretization matrix in (2.8), only direct neighbors are involved, i.e., boxes which have a face in common. Thus, it is fairly reasonable to compare the FVA only with methods whose stencils are defined on a box discretization or on its dual grid and, even more importantly, solely involve direct neighbors. Therefore we confine ourselves to finite differences methods with accuracy order no more than two and excludes, e.g., finite element methods as well as finite difference and finite volume methods of higher order of accuracy.

In our numerical experiment, we consider a two-well potential in two dimensions,

$$
V(x, y)=\frac{5}{2}\left(1-x^{2}\right)+5 y^{2},
$$

on a rectangular domain $\Omega=[-1,1] \times[-0.8,0.8]$. The contour plot of the potential landscape is given in the left panel of Figure 3.1. If the sets $A$ and $B$ are chosen as

$$
A=\{(x, y): x<-0.8\} \cap \Omega \text { and } B=\{(x, y): x>0.8\} \cap \Omega,
$$

then the committor equation in (3.1) admits the analytical solution [10]

$$
\left.q(x, y)=\left(\int_{-0.8}^{0.8} e^{\frac{5}{2}\left(1-z^{2}\right)^{2}} d z\right)\right)^{-1} \int_{-0.8}^{x} e^{\frac{5}{2}\left(1-z^{2}\right)^{2}} d z
$$

that is shown in the right panel of Figure 3.1. Notice that the exact committor function only depends on the coordinate $x$ (because the sets $A$ and $B$ do) and it can be sufficiently accurately computed using quadrature.

The exact committor function allows for numerically computing the order of accuracy of our scheme that is expected to be of second order. To this end the committor equation is numerically solved with the finite volume scheme on a sequence of grids on $\Omega_{A, B}$, where each grid consists of $M^{2}$ equal sized boxes (cells) for $M=20, \ldots, 200$ and covers the set $\Omega \backslash(A \cup B)$. Notice that the Neumann boundary condition in (3.1),

$$
\nabla q \cdot \boldsymbol{n}=0
$$



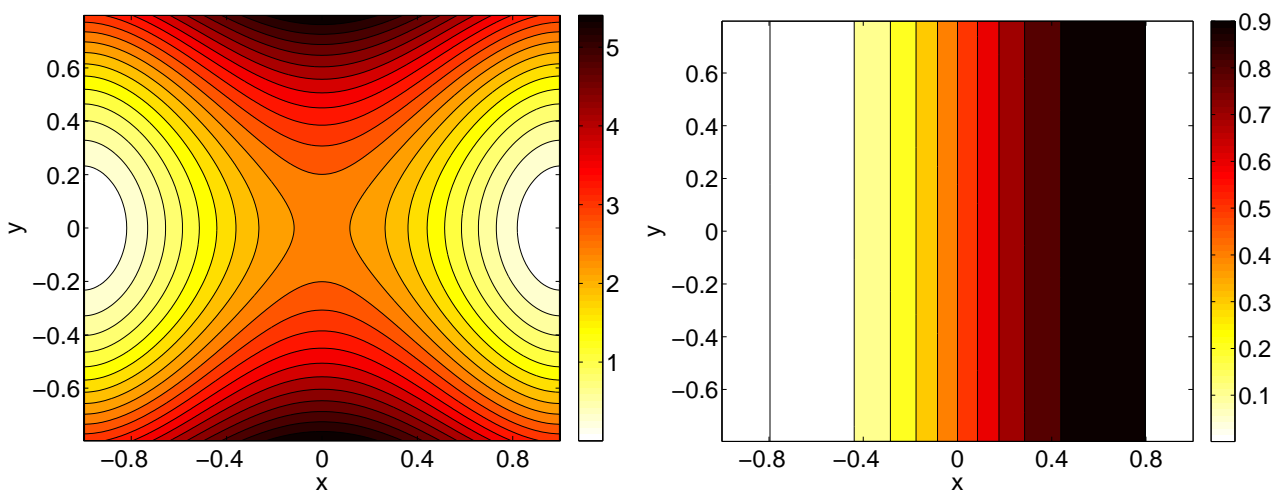

Figure 3.1. Left panel: The contour plot of the two-well potential given in (3.2) on the domain $\Omega=[-1,1] \times[-0.8,0.8]$. Right panel: The level sets of the exact committor function $q(x, y)$ given in (3.3) for the inverse temperature $\beta=1$. The value $q(x, y)$ admits a probabilistic interpretation; it is the probability that the process starting in $(x, y)$ reach the set $A=\{(x, y): x<-0.8\} \cap \Omega$ first rather than the set $B=\{(x, y): x>0.8\} \cap \Omega$.

would directly translate into a zero entry in the discretization matrix $\boldsymbol{A}$ if the cell under consideration had a neighbor cell in the direction of $\boldsymbol{n}$ (cf. Equation 2.11). Consequently, unlike, e.g., in the method of finite differences, the discretization stencil for a boundary cell $\Omega_{i}$ is unaffected in the sense that the entries $\boldsymbol{A}_{i j}$ corresponding to neighbor cells remain the same (cf. (2.8)). In other words, the detail balance condition (2.14) with respect to the discrete Boltzmann distribution is invariant under imposing Neumann-boundary conditions. Furthermore, observe that neglecting a cell in the discretization directly corresponds to imposing Neumann boundary conditions on the neighbored cells.

The numerical error $\|q-\hat{q}\|_{\infty}$ between the exact committor function $q$ evaluated at the box centers and the numerical solution $\hat{q}$ is depicted in Figure 3.2 as a function of the box width (here: in the $x$-direction, $h_{x}$ ). The double logarithmic plot reveals the second order accuracy of our scheme. Additionally, the committor equation is numerically solved with standard finite differences schemes [17] on the grid spanned by the box centers of the grid. In particular, the finite volume scheme is compared to a central differences scheme (resulting from a second order discretization of the gradient term in $\mathcal{L}$ via central differences) and an upwind scheme (resulting from a first order upwind discretization of $\mathcal{L}$ ). It can be seen that the finite volume scheme clearly has a smaller error than the finite differences schemes.

3.2. Application to Lennard-Jones cluster rearrangement. The purpose of this second example is to illustrate that the FVA is more than "simply" a discretization method by demonstrating the interplay between our discretization scheme and discrete Transition Path Theory (TPT), a recently introduced method to investigate rare events in Markov jump processes, i.e., transitions between metastable regions. More specifically, we analyze the rearrangement of a Lennard-Jones cluster in the plane via discrete TPT by exploiting the fact that the discretization matrix $\boldsymbol{A}$ can be interpreted as the infinitesimal generator of a MJP. By construction, the MJP is reversible with respect to the (discrete) Boltzmann distribution and, hence, it is a discrete analog of the reversible diffusion process (discrete TPT heavily relies on this property). The strategy to analyze rare events which we will describe in this section 


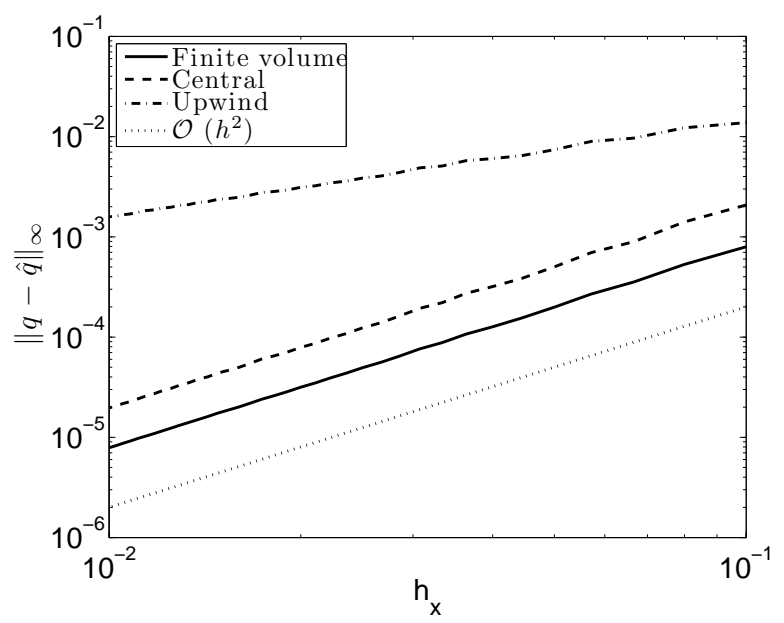

Figure 3.2. Numerical error $\|q-\hat{q}\|_{\infty}$ between the exact committor function $q$ and the $n u$ merical solution $\hat{q}$ of the committor Equation (3.1) as a function of the spatial discretization in the $x$-direction, $h_{x}$.

has successfully been applied to detect mechanisms of protein-ligand association and its modulation by protein mutations [19].

3.2.1. Transition Path Theory. Transition Path Theory is concerned with transitions in Markov processes and has first been developed in [10, 41, 30] in the context of diffusion processes, in particular for systems of the form in (1.1). The basic idea is to single out two disjoint subsets in state space, say $A, B \subset \Omega$, and determine the "preferred" mechanism by which the dynamics makes a transition (reaction) from $A$ to $B$. Typically the sets $A$ and $B$ are metastable regions representing, e.g., conformations of biomolecules [38]. Transitions between metastable regions are rare events and therefore any sampling-based method for the detection of transitions mechanisms and transition rates would cause an enormous numerical effort.

TPT goes beyond sampling in that the underlying committor function completely encodes the statistical properties of the ensemble of all reactive trajectories (transitions) can be efficiently computed by solving a mixed boundary value problem in (3.1). TPT provides expressions for the probability distribution of reactive trajectories, the associated probability current and flux, and the corresponding transition rates. In applications one is often interested in identifying the most probable transition mechanism from $A$ to $B$, i.e, in the region in state space through which the most transitions happen per unit of time. Accordingly the transition tubes are characterized by the current of reactive trajectories. Accurately solving the PDE (3.1) is of course impossible if the problem's dimension is high. The remedy then is discrete TPT - Transition Path Theory for Markov jump processes - a generalization of TPT to the Markov jump processes [31]. Within the discrete TPT framework the reactive properties of a system are captured in a discrete transition network and the preferred transition pathways can effectively computed via graph algorithms [29].

3.2.2. Lennard-Jones cluster. Inspired by the considerations in [8, 44], we will take a look at the rearrangement of a cluster of three particles in the plane whose dynamics is governed by the Smoluchowski dynamics (1.1). The interaction of the 
particles is described by the Lennard-Jones potential

$$
V_{L J}\left(\mathbf{x}^{1}, \mathbf{x}^{2}, \mathbf{x}^{3}\right)=\sum_{1 \leq i<j \leq 3} 4 \epsilon\left[\left(\frac{\sigma}{\left|\mathbf{x}^{i}-\mathbf{x}^{j}\right|}\right)^{12}-\left(\frac{\sigma}{\left|\mathbf{x}^{i}-\mathbf{x}^{j}\right|}\right)^{6}\right],
$$

where $\mathbf{x}^{i} \in \mathbb{R}^{2}, i=1,2,3$ denotes the position of $i$-th particle. The potential assumes its minimum at

$$
\left(\mathbf{m}^{1}, \mathbf{m}^{2}, \mathbf{m}^{3}\right) \in\left\{\left(\mathbf{x}^{1}, \mathbf{x}^{2}, \mathbf{x}^{3}\right):\left\|\mathbf{x}^{i}-\mathbf{x}^{j}\right\|_{2}=2^{1 / 6} \sigma, 1 \leq i<j \leq 3\right\},
$$

with $V\left(\mathbf{m}^{1}, \mathbf{m}^{2}, \mathbf{m}^{3}\right)=-3 \epsilon$. Geometrically this minimizer corresponds to a triangular arrangement of the three particles with constant distance $r_{\min }=2^{1 / 6} \sigma$. Although the potential is invariant under translation and rotation, we assume that the particles are distinguishable. Therefore there are two essentially different equilibrium configurations as is schematically illustrated in Figure 3.3. In the following we refer to the left configuration in the figure as $1-2-3$ and to the right one as $1-3-2$.

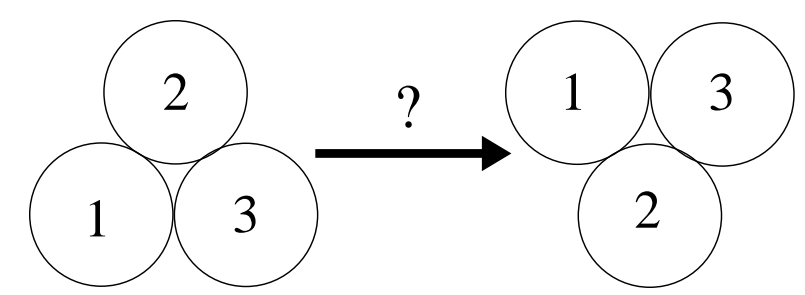

FIGURE 3.3. Schematic illustration of the two different equilibrated configurations of the three particle Lennard-Jones cluster in the plane. The interaction of the particle is described by the potential in (3.4). We are interested in the rearrangement of the left configuration (denoted by $1-2-3)$ to the right configuration (denoted by 1-3-2) under the Smoluchowski dynamics.

The question now is the following: what is the preferred rearrangement of the cluster starting in the $1-2-3$ configuration and ending up in the $1-3-2$ one, and how does the preferred transition path changes as temperature is varied?

Formulated in the language of discrete TPT, we ask what is the dominant reaction pathway from $1-2-3(\operatorname{set} A)$ to $1-3-2(\operatorname{set} B)$ ? Notice that the rearrangement from the configuration $1-2-3$ to $1-3-2$ is non-trivial because it cannot be described by translation or rotation. This, together with the fact that the interaction potential is invariant under translations and rotations, allows us to reduce the dimensionality of the problem by restricting the motion of the Lennard-Jones cluster as follows. First, we fix particle one in the origin and, secondly, we restrict the movement of particle three to the $x$-axis of the plane. The dynamics of the restricted cluster are then governed by a three-dimensional potential landscape of the form

$$
V\left(x_{1}^{2}, x_{2}^{2}, x_{1}^{3}\right) \stackrel{\text { def }}{=} V_{L J}\left((0,0),\left(x_{1}^{2}, x_{2}^{2}\right),\left(x_{1}^{3}, 0\right)\right) .
$$

The corresponding MJP as a discrete analog of (1.1) results from a coarse $30 \times 30 \times$ 30 box discretization of the rectangular domain $\Omega=\left[0, r_{\text {cut }}\right] \times\left[-r_{\text {cut }}, r_{\text {cut }}\right] \times\left[0, r_{\text {cut }}\right]$ with $r_{c u t}=2.5 \sigma \approx 2.22 \cdot 10^{-1}$. For the well-depth $\epsilon=5$, the configurations $1-2-3$ and $1-3-2$ are metastable configurations. To ensure that the MJP is irreducible - at least for all practical purposes — only boxes with a potential energy below 
a prescribed threshold are considered. In other words, grid points with too high potential energy (compared to the minimum $-3 \epsilon$ ) are negligible as their invariant measure is vanishingly small and henceforth omitted.

REMARK 3.1. Notice that the detailed balance relation is unaffected by neglecting boxes (with high potential energy), since neglecting a box imposes reflecting boundary conditions, i.e. Neumann-boundary conditions, on the neighbored boxes (cf. Sect. 3.1).

In principle only two different transition mechanisms are possible for the transition from the $1-2-3$ to the $1-3-2$ configuration; either particle two moves down on a vertical straight line while particle three is sidestepping, or particle three remains fixed while particle two is circumventing. Notice that the symmetric case, i.e., circumventing particle one, is excluded by the choice of $\Omega$. The computation of the dominant reaction pathway via discrete TPT shows that at high temperature, $\beta=1$, the first mechanism is preferred, whereas at low temperature, $\beta=5$, the second one is preferred. For a schematic illustration of the pathways see Figure 3.4. From a physical point of view this behavior is reasonable as the path through the "middle" is admissible only if particle three is far away enough from particle one, so that particle two can slip through. At high temperature this scenario is relatively probable, for particle three moves rapidly back and forth and there is a "finite window" for particle two to slip through. However the window becomes smaller and smaller the lower temperature gets. Therefore the dynamics prefers the circumventing route at low temperature since it is not conditioned by the position of particle number three.

A mathematical argument for the validity of the explanation is given by the theory of Freidlin and Wentzell [13], which states that at low temperature the dynamics prefers the transition pathways along the lowest energy barriers; the potential energies of the two respective dominant reaction paths is depicted in Figure 3.4. As indicated by the dashed lines, the highest barrier at low temperature (left panel) is indeed less than the one at high temperature (right panel). Besides the preferred reaction pathway, discrete TPT gives us the transition rate $k_{A B}$, i.e., the average number of transitions from $A$ to $B$ per unit of time. Note that at low temperature, $\beta=5$, the transition rate between these metastable states is $k_{A B}=2.9 \cdot 10^{-8}$. The numerical approximation of the governing dynamics presented in this article makes it possible to apply TPT for Markov jump processes and thus find these pathways.

3.3. Sampling the invariant distribution. In many applications (e.g., proteins dynamics $[12,14,20])$ DNS, by means of the classical Euler-Maruyama scheme, is the method of choice, although it is well known that it is biased and, specifically, does not preserve reversibility (for details see Section 3.3.2).

This last example is devoted to propose the FVA as an alternative method for sampling stochastic processes of the form (1.1). Once again, the basic idea is to interpret the discretization matrix $\boldsymbol{A}$ as an infinite generator of a MJP which i) is a discrete analog of the original process and ii) preserves reversibility with respect to the approximated Boltzmann measure. Consequently, drawing sample paths from the MJP allows for the exploration of the state space in order to, e.g., compute observables of interest such as free energy or to detect metastable regions. For instant, in the context of reaction kinetics, this sampling approach is well known as the Gillespie algorithm [16] and has been successfully applied to study high dimensional reactions.

To assess FVA as an numerical sampling scheme, we compare it to standard schemes, here to the prominent Euler-Maruyama scheme. In the remainder of this 

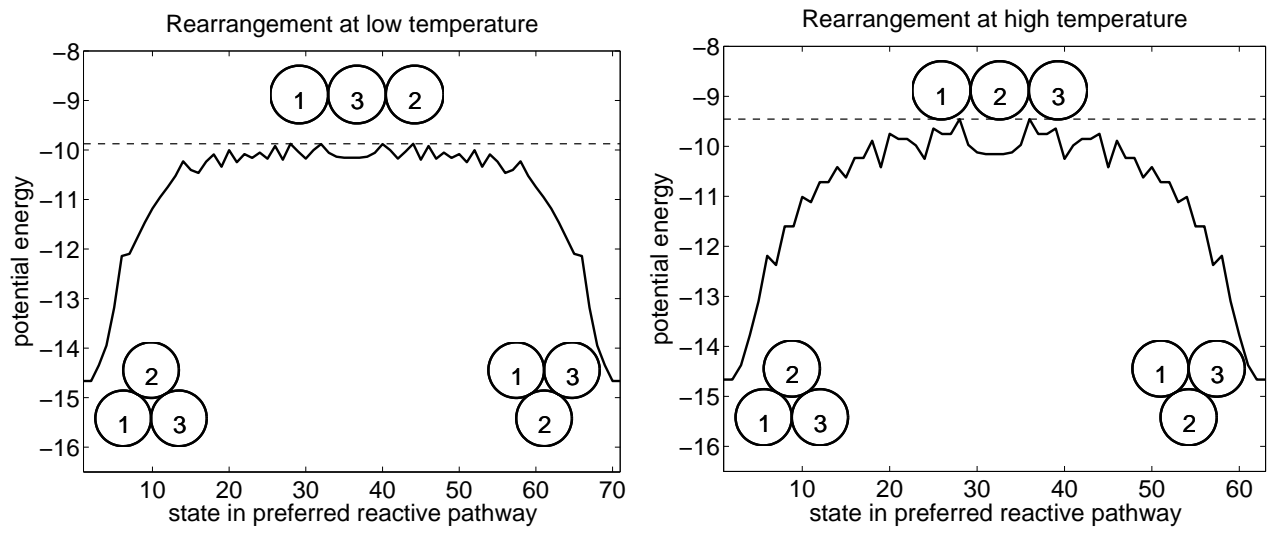

Figure 3.4. Potential energy along the reaction pathway from the initial cluster configuration $1-2-3$ to the final cluster configuration $1-3-2$. Left panel: Energy profile for the low temperature $\beta=5$. Right panel: Energy profile for the high temperature $\beta=1$. Results are shown for $\epsilon=5$ and a $30 \times 30 \times 30$ box discretization of the rectangular domain $\Omega=\left[0, r_{\text {cut }}\right] \times\left[-r_{\text {cut }}, r_{\text {cut }}\right] \times\left[0, r_{\text {cut }}\right]$ with $r_{\text {cut }}=2.5 \sigma \approx 2.22 \cdot 10^{-1}$.

section, we explain in detail such a comparison by sampling the invariant distribution of a low dimensional potential landscape. Particularly, we elaborate on how to compare a sampling scheme based on a spatial discretization with a scheme arising from a discretization in time. Regarding the application to high dimensional dynamics, we also comment on the computational cost of the FVA scheme.

3.3.1. Sampling from MJP. To sample the stationary distribution of the SDE (1.1) we exploit the characterization of the matrix $\boldsymbol{A}$ as the infinitesimal generator of a Markov jump process (MJP) on the discrete state space $S$ which i) is a discrete analog of the original process and ii) preserves reversibility with respect to the approximated Boltzmann measure. The idea now is to draw a realization from the MJP, i.e., a sequence $\left(i_{1}, t_{i_{1}} ; i_{2}, t_{i_{2}} ; \ldots ; i_{N}, t_{i_{N}}\right)$ of states $i_{k} \in S$ and residence times $t_{i_{k}}$ that the process spends in the state $i_{k}$ before jumping to the next state $i_{k+1}$. A realization of a MJP with generator $\boldsymbol{A}$ can be generated by the following iteration [34]: Suppose the MJP starts in $i_{0} \in S$. Then

1. draw a resident time $t_{i_{k}}$ according to

$$
t_{i_{k}}=\log (u)\left(A_{i_{k}, i_{k}}\right)^{-1}
$$

with $u$ uniformly distributed on $[0,1]$,

2. draw the next state $i_{k+1}$ with probability

$$
\mathbb{P}\left[i_{k+1}=j\right] \propto A_{i_{k}, j} \quad j \neq i_{k} .
$$

3.3.2. Convergence to the invariant measure. Now we may ask how fast does our scheme sample the invariant measure and how does it perform compared to a standard integrator like Euler-Maruyama. Suppose that at time $t=0$ the MJP is distributed according to the initial probability distribution $\boldsymbol{\nu}$. Further let $\boldsymbol{p}(t)$ be the distribution at time $t \geq 0$ that is governed by the master equation [5],

$$
\frac{d \boldsymbol{p}(t)}{d t}=\mathbf{A}^{T} \boldsymbol{p}(t), \quad \boldsymbol{p}(0)=\boldsymbol{\nu}
$$


By construction, the invariant measure of our MJP $\boldsymbol{\pi}$ solves $\boldsymbol{A}^{T} \boldsymbol{\pi}=0$. Given a uniform grid on $\Omega$ the vector $\pi$ has the elements

$$
\pi_{i}=Z^{-1} \exp \left(-\beta V\left(x_{i}\right)\right), \quad i \in S,
$$

with $Z^{-1}$ being the normalization constant and $x_{i}$ being the center of cell $\Omega_{i}$. Reversibility implies that the MJP converges exponentially to its invariant measure. More precisely, let $0=\lambda_{1}>\lambda_{2} \geq \lambda_{3} \geq \ldots \geq \lambda_{|S|}$ be the eigenvalues of A. By reversibility, all eigenvalues are real and the following estimate holds [32, 33]:

$$
\|\boldsymbol{p}(t)-\boldsymbol{\pi}\|_{1} \leq K e^{\lambda_{2} t}, \quad t \geq 0,
$$

where $\|\cdot\|_{1}$ is the discrete $l_{1}$-norm and $K>0$ is a constant depending on $\mathbf{A}$.

Conversely for SDEs of the type (1.1), applying the Euler-Maruyama scheme (see, e.g., [22]) yields an time-discrete iteration of the form

$$
\hat{X}_{k+1}=\hat{X}_{k}-\nabla V\left(\hat{X}_{k}\right) \tau+\sqrt{2 \beta^{-1} \tau} \eta
$$

where $\hat{X}_{k}$ denotes the time-discrete approximation of $X_{t_{k}}$ with $\hat{X}_{0}=X_{0}, \tau=t_{k+1}-t_{k}$ is the integration time step, and $\eta$ is a $\mathcal{N}(0,1)$ Gaussian random variable.

For a general, multi-well potential $V$, sampling the Boltzmann distribution using (3.5) requires exponentially long trajectories. Therefore the time discretization inevitably introduces a bias in the invariant distribution of the SDE, even as $\tau \rightarrow 0$. Even worse, if the gradient field $\nabla V$ is non-globally Lipschitz then the Euler-Maruyama scheme is transient for all $\tau>0$ with unbounded moments [28]. The bias can be removed by augmenting the iteration (3.5) with an additional Metropolis-Hastings acceptance step, thereby using the Euler iteration merely as a proposal generator for a Monte-Carlo sampler [37, 4]. Using an approximation to the true dynamics for generating proposals rather than completely random moves guarantees that the proposals are "physically sensible" and the rejection rate is low. Moreover the scheme converges with probability one to the correct Boltzmann distribution for any stable step size $\tau>0$.

3.3.3. On how to compare MJP with Euler-Maruyama. Comparing a MJP with the Euler-based Monte-Carlo scheme sounds like comparing apples and oranges, for Euler-Maruyama is a time discretization method, whereas our approach is based on a spatial discretization of the infinitesimal generator. In addition, the Euler-Maruyama scheme is stochastic in a continuous state space, whereas the MJP is stochastic in a discrete state space and stochastic in time. In the first case, moreover, the state space is unbounded. Hence, for a comparison of both schemes, the process is restricted to a bounded domain $\Omega \subset \mathbb{R}^{n}$ (here: $n=2$ ) where restriction means that $\Omega$ is chosen such the probability to find the equilibrated process in regions close to the boundary of $\Omega$ is of the order of the machine accuracy.

Now let $S=\left\{\Omega_{1}, \ldots, \Omega_{M^{2}}\right\}$ be a grid (box discretization) covering the domain $\Omega \subset \mathbb{R}^{2}$ with $M^{2}$ equal sized boxes. The invariant distribution of the SDE restricted on the boxes is then given by the discrete Boltzmann distribution

$$
\hat{\boldsymbol{\pi}}=\left(\hat{\pi}_{1}, \ldots, \hat{\pi}_{M^{2}}\right), \quad \hat{\pi}_{i}=Z^{-1} \int_{\Omega_{i}} \exp (-\beta V(x, y)) d x d y,
$$

with $Z$ being a normalization constant. Although the exact stationary distribution of the corresponding MJP is different, namely,

$$
\boldsymbol{\pi}=\left(\pi_{1}, \ldots, \pi_{M^{2}}\right), \quad \pi_{i} \propto \exp \left(-\beta V\left(x_{i}, y_{j_{i}}\right)\right),
$$


we may still compare the MJP and the Euler-Maruyama scheme in terms of their convergence to $\hat{\boldsymbol{\pi}}$, for the difference between $\boldsymbol{\pi}$ and $\hat{\boldsymbol{\pi}}$ is negligible.

We measure the convergence of either method by the $l_{1}$-norm,

$$
\mathbb{E}\left[\|\hat{\boldsymbol{\pi}}-\tilde{\boldsymbol{p}}(T)\|_{1}\right],
$$

where $\tilde{\boldsymbol{p}}(T)=\left(\tilde{p}_{1}(T), \ldots, \tilde{p}_{M^{2}}(T)\right)$ denotes the state probability distribution resulting from a finite realization of the MJP or the Euler-Maruyama scheme at time $T>0$. To be more precise, the state probability distribution, $\tilde{\boldsymbol{p}}(T)$, associated with a realization of the MJP, $\left(i_{0}, t_{i_{0}} ; \ldots ; i_{N}, t_{i_{N}}=T\right)$, is computed by the time average

$$
\hat{p}_{j}(T)=\frac{1}{T} \sum_{k=0}^{N} t_{i_{k}} \delta_{j, i_{k}} \quad \forall j \in S,
$$

whereas the distribution of the Metropolis-adjusted Euler scheme is computed from a normalized histogram whose bins are defined by the cells $\Omega_{i}$.

In order to make the convergence results of both methods comparable, the simulations are coupled such that they require the same total numerical effort. To understand the following algorithmic procedure it is helpful to bear in mind that we are interested in computing expectation values. Therefore we generate an ensemble consisting of $L>0$ trajectories with a total time $T>0$ from either method and approximate the expectation value by averaging over the different realizations. Furthermore it is important to realize that the number of jumps $N$ of a MJP-trajectory of total time $T>0$ is random, and hence the total numerical effort of generating $L$ trajectories,

$$
C_{M J P}=\sum_{i=1}^{L} N_{i},
$$

is random too. Consequently, choosing $N_{\text {Euler }}=C_{M J P} / L$ and $\tau=T / N_{\text {Euler }}$ in the Metropolis-adjusted Euler-Maruyama scheme leads to the same total numerical effort as for the MJP scheme. The numerical experiment is summarized as follows:

1. For a fixed total time $T>0$, generate $L>0$ MJP-trajectories.

2. Approximate the expectation in (3.6) by running averages of $\|\hat{\boldsymbol{\pi}}-\tilde{\boldsymbol{p}}(T)\|_{1}$.

3. Generate $L>0$ trajectories with the Metropolis-Euler-Maruyama scheme of constant length $N_{\text {Euler }}=C_{M J P} / L$ and constant time step $\tau=T / N_{\text {Euler }}$.

4. Approximate the expectation in (3.6) by running averages.

Before we present our numerical experiments, we discuss and compare the computational complexity of both methods in terms of potential evaluations, respectively. First of all, both schemes are one-step schemes because the next sample is computed from the current sample in one step. The evaluation of the gradient in the EulerMaruyama scheme (usually done by finite differences) involves $2 d+1$ evaluations of the potential plus drawing of $d$ univariate Gaussian random variables. The computational cost of the FVA scheme is then comparable to that of the Euler-Maruyama scheme, since jumping to a new state of the MJP involves drawing two uniform random variables and $2 d+1$ evaluations of the potential. It is important to notice that no explicit spacial discretization is needed for sampling via $\mathrm{FVA}^{2}$. We conclude that

\footnotetext{
${ }^{2}$ That is, the mesh and the transition matrix $\boldsymbol{A}$ of the MJP do not need to be computed and stored a priori, but instead at each step the transition rates to the neighboring mesh points can be computed on the fly.
} 
the computational cost of one step in both methods scales linearly with the dimension $d$. In the next section we give numerical evidence that the overall performance of both schemes is comparable as well.

3.3.4. Numerical results. As a potential in (1.1) we choose the 2dimensional three-well potential

$$
\begin{aligned}
V(x, y)= & 3 e^{-x^{2}-\left(y-\frac{1}{3}\right)^{2}}-3 e^{-x^{2}-\left(y-\frac{5}{3}\right)^{2}} \\
& -5 e^{-(x-1)^{2}-y^{2}}-5 e^{-(x+1)^{2}-y^{2}}+\frac{1}{5} x^{4}+\frac{1}{5}\left(y-\frac{1}{3}\right)^{4}
\end{aligned}
$$

that is a well-known test example for studying rare events (see, e.g., [21, 35, 31]). As the left panel of Figure 3.5 shows, the potential (3.8) has two deep minima approximately at $( \pm 1,0)$, a shallow minimum approximately at $(0,1.5)$, three saddle points approximately at $( \pm 0.6,1.1),(-1.4,0)$ and a maximum at $(0,0.5)$.
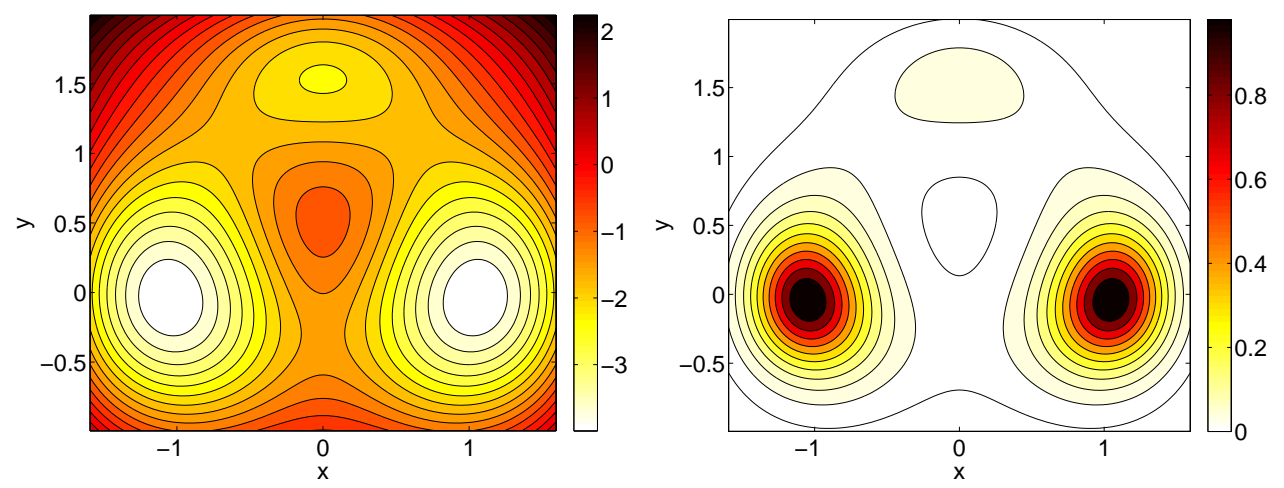

Figure 3.5. Left panel: Contour plot of the three-well potential in (3.8). Right panel: The invariant distribution, $\exp (-\beta V)$, of the Markov process in (1.1) for the inverse temperature $\beta=1.67$ indicates metastability.

For the inverse temperature $\beta=1.67$, the invariant distribution $\mu \propto \exp (-\beta V)$ is shown in the right panel of Figure 3.5. The sharp peaks located at the minima of the potential indicate that the dynamics (1.1) are metastable, i.e., the process spends a long time in the vicinity of a minimum before it makes a transition to another well. In other words, the process exhibits a slow time scale which prevents the process from fast equilibrating, implying slow convergence of any sampling procedure.

The convergence of the schemes is compared using three differently sized grids, $M=20,40,60$, each covering the same rectangular domain $\Omega=[-2,2] \times[-1.5,2.5]$; for a detailed description of the numerical experiment see the previous section. The discrete Boltzmann distribution is sufficiently accurately computed on these grids by using quadrature. The expected numerical error in (3.6) is approximated by an average, $\left\langle\|\hat{\boldsymbol{\pi}}-\hat{\boldsymbol{p}}(T)\|_{1}\right\rangle$, computed from $L=1000$ realizations where each realization starts in the same state, namely, in the center of the cell covering the point $(-1,0)$ being the center of the left minimum.

The average $l_{1}$-error as a function of the total time $T=10000,20000, \ldots, 100000$ is illustrated in Figure 3.6. As one can see, the convergence of the methods considerably differs on the coarsest grid (see left panel). The double logarithmic plot reveals geometric convergence of the metropolized Euler-Maruyama scheme with rate 1/2, 

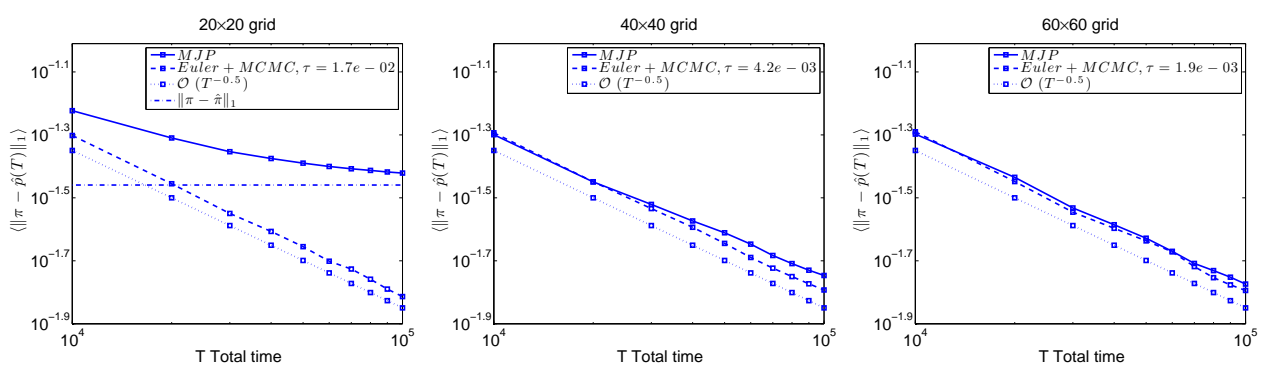

FIGURE 3.6. Convergence of the schemes with respect to the discrete Boltzmann distribution $\hat{\pi}$ is illustrated by the average $l_{1}$-error as a function of the total time $T=10000,20000, \cdots, 100000$. The MJP (solid line) is compared to Metropolis-Euler-Maruyama scheme (dashed lines) using three differently sized grids with $M=20,40,60$.

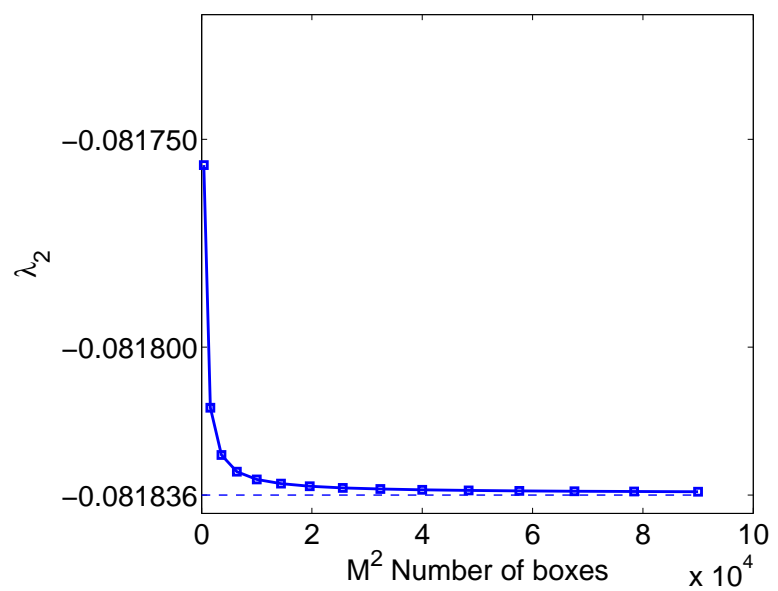

FIGURE 3.7. The graph shows the first nontrivial eigenvalue, $\lambda_{2}<0$, of the generator matrix $\boldsymbol{A}$ (defined in (2.8) as a function of the total number of discretization boxes. The quick convergences indicate that the MJP, even for a coarse grid well, captures the essential dynamics of the underlying SDE. Results for $M=20,40, \ldots, 300$ and $\beta=1.67$.

but the MJP converges at a slower rate. Even worse, the average $\left\langle\|\hat{\boldsymbol{\pi}}-\tilde{\boldsymbol{p}}(T)\|_{1}\right\rangle$ seems to be bounded from below by a constant. Explaining this behavior is simple: the lower bound (as indicated by the dashed line) is the error $\|\boldsymbol{\pi}-\hat{\boldsymbol{\pi}}\|_{1}$. The graph particularly shows that the observable $\tilde{\boldsymbol{p}}(T)$ is almost converged at the final time $T=100000$. The plots in the middle and in the right panel reveal that the convergence on a finer but still coarse grid $(M=40,60)$ is comparable with the convergence of the Metropolisadjusted Euler-Maruyama scheme (which is also reversible).

Next we turn our attention to the first non-trivial eigenvalue, $\lambda_{2}<0$, of the generator matrix $\boldsymbol{A}$ as a function of the total number of discretization boxes. The eigenvalue $\lambda_{2}$ indicates the slowest time scale of the MJP which, here, is the transition process between the two metastable regions in the three-well potential (cf. Fig. 3.5). ${ }^{3}$ As

\footnotetext{
${ }^{3}$ Notice that the numerical computation of the eigenvalues and vectors of the generator matrix $\boldsymbol{A}$ is well-conditioned since $\boldsymbol{A}$ is algebraically similar to a symmetric matrix, $\operatorname{diag}\left(\boldsymbol{\pi}^{1 / 2}\right) \boldsymbol{A} \operatorname{diag}\left(\boldsymbol{\pi}^{-1 / 2}\right)$ with $\boldsymbol{\pi}$ being the stationary distribution of the MJP.
} 
one can see in Figure 3.7, the eigenvalue $\lambda_{2}$ quickly converges as the number of the boxes increases $(M=20,40,60, \ldots, 300)$. Particularly, the graph shows that even for a coarse discretization, e.g., $M=40$, the essential dynamics of the SDE, i.e., the hopping between the two major metastable sets is well captured by the MJP.

\section{Conclusion and outlook}

In this paper we have developed a numerical algorithm based on a finite volume discretization of the infinitesimal generator $\mathcal{L}$ of stochastic differential equations of Smoluchowski type. The resulting discretized operator, $\boldsymbol{A}$, can be interpreted as the infinitesimal generator (rate matrix) of a Markov jump process (MJP) on the chosen grid. By simulating the jump process one can then generate trajectories on the discretized state space (as opposed to discrete-time trajectories as in standard numerical discretization of SDEs). We have shown that our numerical method preserves important properties associated with the original continuous process such as the invariant measure, the forward-backward dichotomy and detailed balance (reversibility), independently of the grid size. Since the discretization preserves detailed balance, the eigenvalues of the discretized infinitesimal generator remain real-valued.

We have also shown with some simple numerical examples that our discretization is suitable for solving boundary value problems as they arise in Transition Path Theory for, e.g., detecting transition mechanisms in molecules, even on a coarse grid. Moreover we have demonstrated that the discrete MJP provides an (ergodic) sampling scheme that allows for computing the discretized Boltzmann distribution without bias, further being exponentially convergent with a rate that is comparable to a Metropolis-adjusted Euler-Maruyama scheme.

There are some issues that arise from any space discretization that we should mention: When the dimension of state space is too large, a spatial discretization by boxes is clearly infeasible. We are currently investigating the possibility of directly computing coarse-grained generator matrices when the system exhibits temporal or spatial scale separation, in which case averaging principles or homogenization theory applies. Here we briefly present the main idea behind this. It is often the case that complex systems present time-scale separation between many fast, irrelevant degrees of freedom which can be "averaged out" in favor of the remaining slow, important directions [27]. Our numerical method preserves the structure of such averaged systems in the sense that the following diagram commutes,

$$
\left\{\begin{array}{lr}
d x_{t}=-\nabla_{x} V\left(x_{t}, y_{t}\right) d t+\sqrt{2 \sigma} d W_{t}, & \text { FVA for full system } \\
d y_{t}=-\frac{1}{\epsilon} \nabla_{y} V\left(x_{t}, y_{t}\right) d t+\sqrt{\frac{2 \sigma}{\epsilon}} d W_{t}, & \dot{\mathbf{u}}=\left(A_{1}+\frac{1}{\epsilon} A_{0}\right) \mathbf{u} \\
\downarrow \text { Averaging for SDE's } & \text { Averaging for MJP } \downarrow \\
d X_{t}=-\nabla_{x} \Psi\left(X_{t}\right) d t+\sqrt{2 \sigma} d W_{t} & \stackrel{\text { FVA for averaged system }}{\longrightarrow}
\end{array}\right.
$$

In the diagram above, $y$ are the fast, high dimensional directions, while $x$ represents the slow and, hopefully, low-dimensional directions which are relevant in the system, and $\epsilon \ll 1$ is the time-scale separation parameter. The effective equation for the averaged dynamics $X_{t}$ involves the computation of the free energy

$$
\Psi(x)=-\beta^{-1} \ln Z(x), \quad Z(x)=\int_{S_{y}} e^{-\beta V(x, y)} d y .
$$

The FVA method preserves this structure in the sense that averaging the full MJP approximation results in a new jump process whose transition rates are precisely 
the equivalent of the transition rates (2.8) for the free energy case. With this in mind, effective coefficients such as free energy, Fixman potential or effective drift and diffusivity (see $[36,23]$ ) can then be recovered and computed consistently from the continuous state process and may be estimated from short simulations of the high dimensional system (e.g., using Monte Carlo sampling). Such an approach is much in the spirit of heterogeneous multiscale methods [9] and will be addressed in a forthcoming article.

A further extension of the finite volume scheme, that we mention here only for completeness, is the formulation of the method on unstructured meshes, e.g., Delaunay triangulation involving an orthogonality condition. In order to avoid the combinatorial explosion in the number of cells in higher dimensions this implies that one needs to derive a priori error estimates as indicated in [3] to allow for adaptive mesh refinement. Eventually, being independent of specific grid geometries would make the method applicable to real world problems such as conformation dynamics of biomolecules.

Acknowledgements. We would like to thank Ralf Kornhuber for valuable discussions about finite volume geometries and for pointing out useful references. We also would like to thank the referees for their constructive criticism which helped us improve this article. This work is supported by the DFG Research Center MATHEON "Mathematics for Key Technologies" (FZT86) in Berlin.

\section{REFERENCES}

[1] D.H. Anderson, Compartmental Modeling and Tracer Kinetics, Lecture Notes in Biomathematics, Springer, Berlin, 50, 1983.

[2] D.N. Arnold, F. Brezzi, B. Cockburn, and L.D. Marini, Unified analysis of discontinuous Galerkin methods for elliptic problems, SIAM J. Numer. Anal., 39(5), 1749-1779, 2002.

[3] R.E. Bank, W.M. Coughran, and L.C. Cowsar, The finite volume Scharfetter-Gummel method for steady convection diffusion equations, Comput. Vis. Sci., 1(3), 123-136, 1998.

[4] N. Bou-Rabee and E. Vanden-Eijnden, Pathwise accuracy and ergodicity of metropolized integrators for SDEs, Commun. Pure Appl. Math., 63(5), 655-696, 2010.

[5] P. Brémaud, Markov Chains: Gibbs Fields, Monte Carlo Simulation and Queues, Springer Verlag, 1999.

[6] F. Brezzi, L.D. Marini, and P. Pietra, Two-dimensional exponential fitting and applications to drift-diffusion models, SIAM J. Numer. Anal., 26(6), 1342-1355, 1989.

[7] Z. Cai, J. Mandel, and S. McCormick, The finite volume element method for diffusion equations on general triangulations, SIAM J. Numer. Anal., 28(2), 392-402, 1991.

[8] C. Dellago, P. Bolhuis, and D. Chandler, Efficient transition path sampling: Applications to Lennard-Jones cluster rearrangements, J. Chem. Phys., 108(22), 9236-9245, 1998.

[9] W. E, B. Engquist, X. Li, W. Ren, and E. Vanden-Eijnden, Heterogeneous multiscale methods: A review, Commun. Comput. Phys., 2(3), 367-450, 2007.

[10] W. E and E. Vanden-Eijnden, Towards a theory of transition paths, J. Statist. Phys., 123(3), 503-523, 2006.

[11] C. Erath and D. Praetorius, A posteriori error estimate and adaptive mesh-refinement for the cell-centered finite volume method for elliptic boundary value problems, SIAM J. Numer. Anal., 47(1), 109-135, 2008.

[12] D.L. Ermak and J.A. McCammon, Brownian dynamics with hydrodynamic interaction, J. Chem. Phys., 69(4), 1352-1360, 1978.

[13] M.I. Freidlin and A.D. Wentzell, Random Perturbations of Dynamical Systems, Springer, New York, 1984.

[14] R.R. Gabdoulline and R.C. Wade, Brownian dynamics simulation of protein-protein diffusional encounter, Methods, 14(3), 329-341, 1998.

[15] C.W. Gardiner, Handbook of Stochastic Methods: For Physics, Chemistry and the Natural Sciences, Springer, 2nd edition, 1996.

[16] D.T. Gillespie, A general method for numerically simulating the stochastic time evolution of coupled chemical reactions, J. Comput. Phys., 22(4), 403-434, 1976. 
[17] W. Hackbusch, Elliptic Differential Equations: Theory and Numerical Treatment, SpringerVerlag, 1992.

[18] U.G. Haussmann and E. Pardoux, Time reversal of diffusions, Ann. Probab., 14(4), 1188-1205, 1986.

[19] M. Held, P. Metzner, J.H. Prinz, and F. Noé, Mechanisms of protein-ligand association and its modulation by protein mutations, Biophys. J. , 100(3), 701-710, 2011.

[20] H.C. Huang and J. M Briggs, The association between a negatively charged ligand and the electronegative binding pocket of its receptor, Biopolymers, 63(4), 247-260, 2002.

[21] S. Huo and J.E. Straub, The maxflux algorithm for calculating variationally optimized reaction paths for conformational transitions in many body systems at finite temperature, J. Chem. Phys., 107(13), 5000-5006, 1997.

[22] P.E. Kloeden and E. Platen, Numerical Solution of Stochastic Differential Equations, SpringerVerlag, 1992.

[23] P.R. Kramer, J.C. Latorre, and A.A. Khan, Two coarse-graining studies of stochastic models in molecular biology, Commun. Math. Sci., 8(2), 482-517, 2010.

[24] J.C. Latorre, Effective Transport Properties of Stochastic Biophysical Models, Doctoral thesis, Rensselaer Polytechnic Institute, 2008.

[25] R.J. LeVeque, Numerical Methods for Conservations Laws, Birkhäuser, Bassel, 1990.

[26] R.J. LeVeque, Finite Volume Methods for Hyperbolic Problems, Cambridge University Press, 2002.

[27] A.J. Majda, I. Timofeyev, and E. Vanden-Eijnden, A mathematical framework for stochastic climate models, Commun. Pure Appl. Math., 54, 891-974, 2001.

[28] J.C. Mattingly, A.M. Stuart, and D.J. Higham, Ergodicity for SDEs and approximations: locally Lipschitz vector fields and degenerate noise, Stoch. Proc. Appl., 101(2), 185-232, 2002 .

[29] P. Metzner, Transition Path Theory for Markov Processes, PhD thesis, Freie Universität Berlin, 2007.

[30] P. Metzner, C. Schütte, and E. Vanden-Eijnden, Illustration of transition path theory on a collection of simple examples, J. Chem. Phys., 125(8), 084110, 2006.

[31] P. Metzner, C. Schütte, and E. Vanden-Eijnden, Transition path theory for Markov jump processes, Multiscale Model. Simul., 7(3), 1192-1219, 2009.

[32] S.P. Meyn and R.L. Tweedie, Markov Chains and Stochastic Stability, Springer, London, 1993.

[33] A. Yu. Mitrophanov, Stability and exponential convergence of continuous-time Markov chains, J. Appl. Probab., 40(4), 970-979, 2003.

[34] J.R. Norris, Markov Chains, Cambridge University Press, 1998.

[35] S. Park, M.K. Sener, D. Lu, and K. Schulten, Reaction paths based on mean first-passage times, J. Chem. Phys., 119(3), 1313-1319, 2003.

[36] G.A. Pavliotis and A.M. Stuart, Multiscale Methods: Averaging and Homogenization, Springer, 2008.

[37] G.O. Roberts and R.L. Tweedie, Exponential convergence of Langevin distributions and their discrete approximations, Bernoulli, 2(4), 341-363, 1996.

[38] Ch. Schütte and W. Huisinga, Biomolecular conformations can be identified as metastable sets of molecular dynamics, in P.G. Ciarlet and C. Le Bris, (eds.), Special Volume: Computational Chemistry, Handbook of Numerical Analysis, Elsevier, X, 699-744, 2003.

[39] N. Singhal, C.D. Snow, and V.S. Pande, Using path sampling to build better Markovian state models: Predicting the folding rate and mechanism of a tryptophan zipper beta hairpin, J. Chem. Phys., 121(1), 415-425, 2004.

[40] J.W. Thomas, Numerical Partial Differential Equations; Finite Difference Methods, Springer, New York, 1995.

[41] E. Vanden-Eijnden, Transition path theory, in M. Ferrario, G. Ciccotti, and K. Binder, (eds.), Computer Simulations in Condensed Matter: from Materials to Chemical Biology, Springer Verlag, 2(703), 2006.

[42] H. Wang, C.S. Peskin, and T.C. Elston, A robust numerical algorithm for studying biomolecular transport processes, J. Theoret. Biol., 221(4), 491-511, 2003.

[43] P. Wesseling, Principles of Computational Fluid Dynamics, Springer, Berlin, 2001.

[44] T. Yanao, W.S. Koon, and J.E. Marsden, Mass-related dynamical barriers in triatomic reactions, Few-Body Systems, 38(2-4), 161-166, 2006. 